\title{
Generalising the scattered property of subspaces
}

\author{
Bence Csajbók, Giuseppe Marino, Olga Polverino and Ferdinando Zullo*
}

July 25,2020

\begin{abstract}

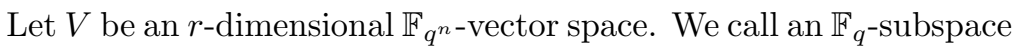
$U$ of $V h$-scattered if $U$ meets the $h$-dimensional $\mathbb{F}_{q^{n} \text {-subspaces of }}$ $V$ in $\mathbb{F}_{q}$-subspaces of dimension at most $h$. In 2000 Blokhuis and Lavrauw proved that $\operatorname{dim}_{\mathbb{F}_{q}} U \leq r n / 2$ when $U$ is 1 -scattered. Subspaces attaining this bound have been investigated intensively because of their relations with projective two-weight codes and strongly regular graphs. MRD-codes with a maximum idealiser have also been linked to $r n / 2$-dimensional 1 -scattered subspaces and to $n$-dimensional $(r-1)$ scattered subspaces.

In this paper we prove the upper bound $r n /(h+1)$ for the dimension of $h$-scattered subspaces, $h>1$, and construct examples with this dimension. We study their intersection numbers with hyperplanes, introduce a duality relation among them, and study the equivalence problem of the corresponding linear sets.
\end{abstract}

\section{Introduction}

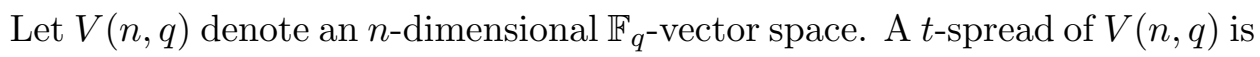
a set $\mathcal{S}$ of $t$-dimensional $\mathbb{F}_{q}$-subspaces such that each vector of $V(n, q) \backslash\{\mathbf{0}\}$ is contained in exactly one element of $\mathcal{S}$. As shown by Segre in [26], a $t$-spread of $V(n, q)$ exists if and only if $t \mid n$.

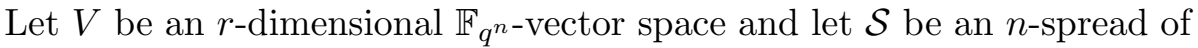
$V$, viewed as an $\mathbb{F}_{q}$-vector space. An $\mathbb{F}_{q}$-subspace $U$ of $V$ is called scattered

\footnotetext{
${ }^{*}$ The research was supported by the Italian National Group for Algebraic and Geometric Structures and their Applications (GNSAGA - INdAM). The first author was partially supported by the János Bolyai Research Scholarship of the Hungarian Academy of Sciences and by OTKA grants PD 132463 and K 124950. The last two authors were supported by the project "VALERE: VAnviteLli pEr la RicErca" of the University of Campania "Luigi Vanvitelli".
} 
w.r.t. $\mathcal{S}$ if it meets every element of $\mathcal{S}$ in an $\mathbb{F}_{q}$-subspace of dimension at most one, see [4]. If we consider $V$ as an $r n$-dimensional $\mathbb{F}_{q}$-vector space, then it is well-known that the one-dimensional $\mathbb{F}_{q^{n} \text {-subspaces of } V \text {, viewed }}$ as $n$-dimensional $\mathbb{F}_{q}$-subspaces, form an $n$-spread of $V$. This spread is called the Desarguesian spread. In this paper scattered will always mean scattered w.r.t. the Desarguesian spread. For such subspaces Blokhuis and Lavrauw showed that their dimension can be bounded by $r n / 2$. After a series of papers, it is now known that when $2 \mid r n$ then there always exist scattered subspaces of this dimension [1, 3, 4, 11.

In this paper we introduce and study the following special class of scattered subspaces.

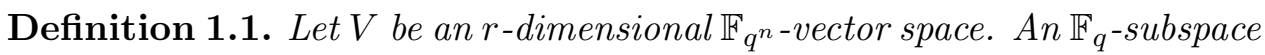
$U$ of $V$ is called h-scattered, $0<h \leq r-1$, if $\langle U\rangle_{\mathbb{F}_{q^{n}}}=V$ and each $h$ -

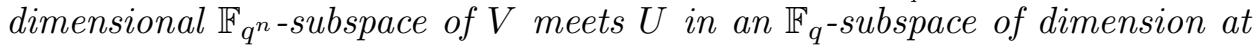
most $h$. An h-scattered subspace of highest possible dimension is called a maximum h-scattered subspace.

With this definition, the 1-scattered subspaces are the scattered subspaces generating $V$ over $\mathbb{F}_{q^{n}}$. With $h=r$ the above definition would give

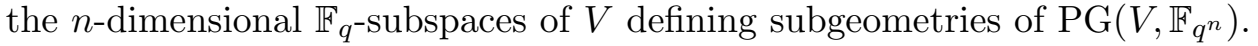
If $h=r-1$ and $\operatorname{dim}_{\mathbb{F}_{q}} U=n$, then $U$ defines a scattered $\mathbb{F}_{q}$-linear set with respect to hyperplanes, introduced in [28, Definition 14]. A further generalisation of the concept of $h$-scattered subspaces can be found in the recent paper [2].

In this paper we prove that for an $h$-scattered subspace $U$ of $V\left(r, q^{n}\right)$, if $U$ does not define a subgeometry, then

$$
\operatorname{dim}_{\mathbb{F}_{q}} U \leq \frac{r n}{h+1},
$$

cf. Theorem 2.3. Clearly, $h$-scattered subspaces reaching bound (1) are maximum $h$-scattered. When $h+1 \mid r$ then our examples prove that maximum $h$-scattered subspaces have dimension $r n /(h+1)$, cf. Theorem 2.6. In Theorem 2.7 we show that $h$-scattered subspaces of dimension $r n /(h+1)$ meet hyperplanes of $V\left(r, q^{n}\right)$ in $\mathbb{F}_{q^{-} \text {-subspaces of dimension at least } r n /(h+1)-n}$ and at most $r n /(h+1)-n+h$. Then we introduce a duality relation between maximum $h$-scattered subspaces of $V\left(r, q^{n}\right)$ reaching bound (1) and maximum $(n-h-2)$-scattered subspaces of $V\left(r n /(h+1)-r, q^{n}\right)$ reaching bound (1), which allows us to give some constructions also when $h+1$ is not a divisor of $r$, cf. Theorem 3.6. 
Proposition 2.1 shows us that $h$-scattered subspaces are special classes of 1-scattered subspaces. In [28, Corollary 4.4] the $(r-1)$-scattered subspaces of $V\left(r, q^{n}\right)$ attaining bound (1), i.e. of dimension $n$, have been shown to be equivalent to MRD-codes of $\mathbb{F}_{q}^{n \times n}$ with minimum rank distance $n-r+1$ and with left or right idealiser isomorphic to $\mathbb{F}_{q^{n}}$. In Section 4 we study the $\mathbb{F}_{q}$-linear set $L_{U}$ determined by an $h$-scattered subspace $U$. In contrast to the case of 1 -scattered subspaces, it turns out that for any $h$-scattered

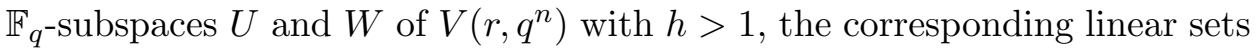
$L_{U}$ and $L_{W}$ are $\operatorname{P\Gamma L}\left(r, q^{n}\right)$-equivalent if and only if $U$ and $W$ are $\Gamma \mathrm{L}\left(r, q^{n}\right)$ equivalent, cf. Theorem 4.5. For $r>2$ this result extends [28, Proposition $3.5]$ regarding the equivalence between MRD-codes and maximum $(r-1)$ scattered subspaces attaining bound (1) into an equivalence between MRDcodes and the corresponding linear sets, see [28, Remarks 4, 5].

\section{The maximum dimension of an $h$-scattered sub- space}

We start this section by the following result.

Proposition 2.1. For $h>1$ the $h$-scattered subspaces are also $i$-scattered for any $i<h$. In particular they are all 1-scattered.

Proof. Let $U$ be an $h$-scattered subspace of $V$. Suppose to the contrary that it is not $i$-scattered for some $i<h$. Therefore, there exists an $i$-dimensional

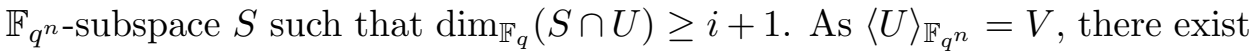
$\mathbf{u}_{1}, \ldots, \mathbf{u}_{h-i} \in U$ such that $\operatorname{dim}_{\mathbb{F}_{q^{n}}}\left\langle S, \mathbf{u}_{1}, \ldots, \mathbf{u}_{h-i}\right\rangle_{\mathbb{F}_{q^{n}}}=h$. Then

$$
\operatorname{dim}_{\mathbb{F}_{q}}\left(U \cap\left\langle S, \mathbf{u}_{1}, \ldots, \mathbf{u}_{h-i}\right\rangle_{\mathbb{F}_{q^{n}}}\right) \geq(i+1)+(h-i)=h+1,
$$

a contradiction.

In the proof of the main result of this section we will need the following lemma.

Lemma 2.2. For any integer $i$ with $r \leq i \leq n$ in $V=V\left(r, q^{n}\right)$ there exists an $(r-1)$-scattered $\mathbb{F}_{q}$-subspace of dimension $i$.

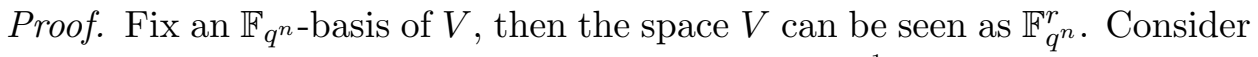
the $n$-dimensional $\mathbb{F}_{q^{\text {-subspace }} U} U=\left\{\left(x, x^{q}, \ldots, x^{q^{r-1}}\right): x \in \mathbb{F}_{q^{n}}\right\}$ of $V$. Let 
$W$ be any $i$-dimensional $\mathbb{F}_{q}$-subspace of $U$. The intersection of $W$ with a hyperplane $\left[a_{0}, a_{1}, \ldots, a_{r-1}\right]$ of $V$ is

$$
\left\{\left(x, x^{q}, \ldots, x^{q^{r-1}}\right): x \in \mathbb{F}_{q^{n}}, \sum_{j=0}^{r-1} a_{j} x^{q^{j}}=0\right\} \cap W,
$$

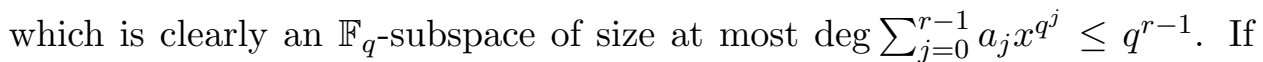
$\langle W\rangle_{\mathbb{F}_{q^{n}}} \neq V$ then there was a hyperplane of $V$ containing $W$, a contradiction, i.e. $W$ is an $(r-1)$-scattered $\mathbb{F}_{q}$-subspace of $V$.

For $h=1$, the following result was shown in [4].

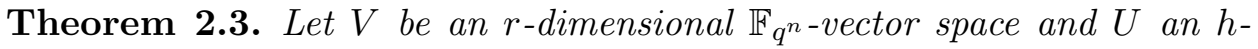
scattered $\mathbb{F}_{q}$-subspace of $V$. Then either

- $\operatorname{dim}_{\mathbb{F}_{q}} U=r, U$ defines a subgeometry of $\mathrm{PG}\left(V, \mathbb{F}_{q^{n}}\right)$ and $U$ is $(r-1)$ scattered, or

- $\operatorname{dim}_{\mathbb{F}_{q}} U \leq r n /(h+1)$.

Proof. Let $k$ denote the dimension of $U$ over $\mathbb{F}_{q}$. Since $\langle U\rangle_{\mathbb{F}_{q^{n}}}=V$, we have $k \geq r$ and in case of equality $U$ defines a subgeometry of $\operatorname{PG}\left(V, \mathbb{F}_{q^{n}}\right)$ which is clearly $(r-1)$-scattered. From now on we may assume $k>r$. First consider

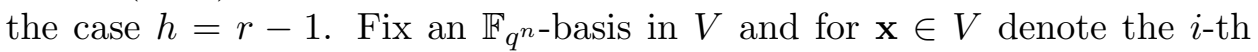
coordinate w.r.t. this basis by $x_{i}$. Consider the following set of $\mathbb{F}_{q}$-linear maps from $U$ to $\mathbb{F}_{q^{n}}$ :

$$
\mathcal{C}_{U}:=\left\{G_{a_{0}, \ldots, a_{r-1}}: \mathbf{x} \in U \mapsto \sum_{i=0}^{r-1} a_{i} x_{i}: a_{i} \in \mathbb{F}_{q^{n}}\right\}
$$

First we show that the non-zero maps of $\mathcal{C}_{U}$ have rank at least $k-r+1$. Indeed, if $\left(a_{0}, \ldots, a_{r-1}\right) \neq \mathbf{0}$, then $\mathbf{u} \in \operatorname{ker} G_{a_{0}, \ldots, a_{r-1}}$ if and only if $\sum_{i=0}^{r-1} a_{i} u_{i}=$ 0, i.e. $\operatorname{ker} G_{a_{0}, \ldots, a_{r-1}}=U \cap H$, where $H$ is the hyperplane $\left[a_{0}, a_{1}, \ldots, a_{r-1}\right]$ of $V$. Since $U$ is $(r-1)$-scattered, it follows that $\operatorname{dim}_{\mathbb{F}_{q}} \operatorname{ker} G_{a_{0}, \ldots, a_{r-1}} \leq r-1$ and hence the rank of $G_{a_{0}, \ldots, a_{r-1}}$ is at least $k-r+1$. Next we show that any two maps of $\mathcal{C}_{U}$ are different. Suppose to the contrary $G_{a_{0}, \ldots, a_{r-1}}=G_{b_{0}, \ldots, b_{r-1}}$, then $G_{a_{0}-b_{0}, \ldots, a_{r-1}-b_{r-1}}$ is the zero map. If $\left(a_{1}-b_{1}, \ldots, a_{r}-b_{r}\right) \neq \mathbf{0}$, then $U$ would be contained in the hyperplane $\left[a_{0}-b_{0}, a_{1}-b_{1}, \ldots, a_{r-1}-b_{r-1}\right]$, a contradiction since $\langle U\rangle_{\mathbb{F}_{q n}}=V$. Hence, $\left|\mathcal{C}_{U}\right|=q^{n r}$.

Suppose to the contrary $k>n$. The elements of $\mathcal{C}_{U}$ form a $n r$-dimensional

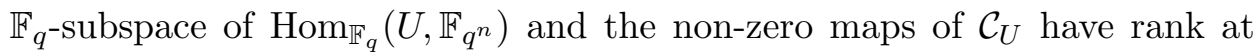


least $k-r+1$. By Result 4.6 (Singleton-like bound) we get $q^{r n} \leq q^{k(n-k+r)}$ and hence $(k-n)(k-r) \leq 0$, which contradicts $k>r$.

From now on, we will assume $1<h<r-1$, since the assertion has been proved in [4] for $h=1$.

First we assume $n \geq h+1$. Then by Lemma 2.2 , in $\mathbb{F}_{q^{n}}^{h}$ there exists an $(h-1)$-scattered $\mathbb{F}_{q}$-subspace $W$ of dimension $h+1$.

Let $G$ be an $\mathbb{F}_{q}$-linear transformation from $V$ to itself with $\operatorname{ker} G=U$. Clearly, $\operatorname{dim}_{\mathbb{F}_{q}} \operatorname{Im} G=r n-k$. For each $\left(\mathbf{u}_{1}, \ldots, \mathbf{u}_{h}\right) \in V^{h}$ consider the $\mathbb{F}_{q^{n} \text {-linear map }}$

$$
\tau_{\mathbf{u}_{1}, \ldots, \mathbf{u}_{h}}:\left(\lambda_{1}, \ldots, \lambda_{h}\right) \in W \mapsto \lambda_{1} \mathbf{u}_{1}+\ldots+\lambda_{h} \mathbf{u}_{h} \in V .
$$

Consider the following set of $\mathbb{F}_{q}$-linear maps $W \rightarrow \operatorname{Im} G$

$$
\mathcal{C}:=\left\{G \circ \tau_{\mathbf{u}_{1}, \ldots, \mathbf{u}_{h}}:\left(\mathbf{u}_{1}, \ldots, \mathbf{u}_{h}\right) \in V^{h}\right\} .
$$

Our aim is to show that these maps are pairwise distinct and hence $|\mathcal{C}|=$ $q^{r n h}$. Suppose $G \circ \tau_{\mathbf{u}_{1}, \ldots, \mathbf{u}_{h}}=G \circ \tau_{\mathbf{v}_{1}, \ldots, \mathbf{v}_{h}}$. It follows that $G \circ \tau_{\mathbf{u}_{1}-\mathbf{v}_{1}, \ldots, \mathbf{u}_{h}-\mathbf{v}_{h}}$ is the zero map, i.e.

$$
\lambda_{1}\left(\mathbf{u}_{1}-\mathbf{v}_{1}\right)+\ldots+\lambda_{h}\left(\mathbf{u}_{h}-\mathbf{v}_{h}\right) \in \operatorname{ker} G=U \text { for each }\left(\lambda_{1}, \ldots, \lambda_{h}\right) \in W .
$$

For $i \in\{1, \ldots, h\}$, put $\mathbf{z}_{i}=\mathbf{u}_{i}-\mathbf{v}_{i}$, let $T:=\left\langle\mathbf{z}_{1}, \ldots, \mathbf{z}_{h}\right\rangle_{q^{n}}$ and let $t=$ $\operatorname{dim}_{q^{n}} T$. We want to show that $t=0$. If $t=h$, then by (2)

$$
\left\{\lambda_{1} \mathbf{z}_{1}+\ldots+\lambda_{h} \mathbf{z}_{h}:\left(\lambda_{1}, \ldots, \lambda_{h}\right) \in W\right\} \subseteq T \cap U,
$$

hence $\operatorname{dim}_{\mathbb{F}_{q}}(T \cap U) \geq \operatorname{dim}_{\mathbb{F}_{q}} W=h+1$, which is not possible since $T$ is an

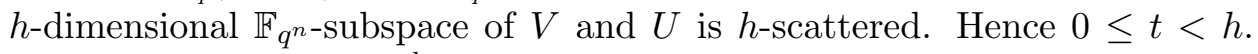
Assume $t \geq 1$. Let $\Phi: \mathbb{F}_{q^{n}}^{h} \rightarrow T$ be the $\mathbb{F}_{q^{n}}$-linear map defined by the rule

$$
\left(\lambda_{1}, \ldots, \lambda_{h}\right) \mapsto \lambda_{1} \mathbf{z}_{1}+\ldots+\lambda_{h} \mathbf{z}_{h}
$$

and consider the map $\tau_{\mathbf{z}_{1}, \ldots, \mathbf{z}_{h}}$. Note that $\tau_{\mathbf{z}_{1}, \ldots, \mathbf{z}_{h}}$ is the restriction of $\Phi$ on the $\mathbb{F}_{q^{\text {-vector }}}$ subspace $W$ of $\mathbb{F}_{q^{n}}^{h}$. It can be easily seen that

$$
\begin{gathered}
\operatorname{dim}_{\mathbb{F}_{q^{n}}} \operatorname{ker} \Phi=h-t, \\
\operatorname{ker} \tau_{\mathbf{z}_{1}, \ldots, \mathbf{z}_{h}}=\operatorname{ker} \Phi \cap W,
\end{gathered}
$$

and by (2)

$$
\operatorname{Im} \tau_{\mathbf{z}_{1}, \ldots, \mathbf{z}_{h}} \subseteq T \cap U
$$




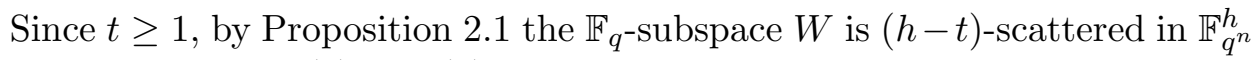
and hence taking (3) and (4) into account we get $\operatorname{dim}_{\mathbb{F}_{q}} \operatorname{ker} \tau_{\mathbf{z}_{1}, \ldots, \mathbf{z}_{h}} \leq h-t$, which yields

$$
\operatorname{dim}_{\mathbb{F}_{q}} \operatorname{Im} \tau_{\mathbf{z}_{1}, \ldots, \mathbf{z}_{h}} \geq t+1
$$

By Proposition 2.1 the $\mathbb{F}_{q}$-subspace $U$ is also a $t$-scattered subspace of $V$, thus by (5)

$$
\operatorname{dim}_{\mathbb{F}_{q}} \operatorname{Im} \tau_{\mathbf{z}_{1}, \ldots, \mathbf{z}_{h}} \leq \operatorname{dim}_{\mathbb{F}_{q}}(T \cap U) \leq t,
$$

contradicting (6). It follows that $t=0$, i.e. $\mathbf{z}_{i}=0$ for each $i \in\{1, \ldots h\}$ and hence $|\mathcal{C}|=q^{r n h}$. The trivial upper bound for the size of $\mathcal{C}$ is the size of $\mathbb{F}_{q}^{(h+1) \times(r n-k)}$, thus

$$
q^{r n h}=|\mathcal{C}| \leq q^{(h+1)(r n-k)},
$$

which implies

$$
k \leq \frac{r n}{h+1} .
$$

Now assume $n<h+1$. By Proposition 2.1 $U$ is $h^{\prime}$-scattered with $h^{\prime}=n-1$. Since $h^{\prime}<r-1$ and $n \geq h^{\prime}+1$, we can argue as before and derive $k=\operatorname{dim}_{\mathbb{F}_{q}} U \leq r n /\left(h^{\prime}+1\right)=r$, contradicting $k>r$.

The previous proof can be adapted also for the $h=1$ case without introducing the subspace $W$, cf. 30].

The following result is a generalisation of [3, Theorem 3.1].

Theorem 2.4. Let $V=V_{1} \oplus \ldots \oplus V_{t}$ where $V_{i}=V\left(r_{i}, q^{n}\right)$ and $V=V\left(r, q^{n}\right)$. If $U_{i}$ is an $h_{i}$-scattered $\mathbb{F}_{q}$-subspace in $V_{i}$, then the $\mathbb{F}_{q}$-subspace $U=U_{1} \oplus$ $\ldots \oplus U_{t}$ is $h$-scattered in $V$, with $h=\min \left\{h_{1}, \ldots, h_{t}\right\}$. Also, if $U_{i}$ is $h$ scattered in $V_{i}$ and its dimension reaches bound (1), then $U$ is h-scattered in $V$ and its dimension reaches bound (1).

Proof. Clearly, it is enough to prove the assertion for $t=2$.

If $h=1$, the result easily follows from Proposition 2.1] and from [3, Theorem 3.1]; hence, we may assume $h=h_{1} \geq 2$.

By way of contradiction suppose that there exists an $h$-dimensional $\mathbb{F}_{q^{n-}}$ subspace $W$ of $V$ such that

$$
\operatorname{dim}_{\mathbb{F}_{q}}(W \cap U) \geq h+1 .
$$

Clearly, $W$ cannot be contained in $V_{1}$ since $U_{1}$ is $h$-scattered in $V_{1}$. Let $W_{1}:=W \cap V_{1}$ and $s:=\operatorname{dim}_{\mathbb{F}_{q^{n}}} W_{1}$. Then $s<h$ and by Proposition 2.1. 
the $\mathbb{F}_{q}$-subspace $U_{1}$ is $s$-scattered in $V_{1}$, thus $\operatorname{dim}_{\mathbb{F}_{q}}\left(U_{1} \cap W_{1}\right) \leq s$. Denoting $\left\langle U_{1}, W \cap U\right\rangle_{\mathbb{F}_{q}}$ by $\bar{U}_{1}$, the Grassmann formula and (7) yield

$$
\operatorname{dim}_{\mathbb{F}_{q}} \bar{U}_{1}-\operatorname{dim}_{\mathbb{F}_{q}} U_{1} \geq h+1-s .
$$

Consider the subspace $T:=W+V_{1}$ of the quotient space $V / V_{1} \cong V_{2}$. Then $\operatorname{dim}_{\mathbb{F}_{q^{n}}} T=h-s$ and $T$ contains the $\mathbb{F}_{q^{\text {-subspace }}}$

$$
M:=\bar{U}_{1}+V_{1}
$$

Since $M$ is also contained in the $\mathbb{F}_{q}$-subspace $U+V_{1}=U_{2}+V_{1}$, then $M$ is $h_{2}$-scattered in $V / V_{1}$ and hence by $h-s \leq h \leq h_{2}$ and by Proposition 2.1. $M$ is also $(h-s)$-scattered in $V / V_{1}$.

On the other hand,

$$
\begin{gathered}
\operatorname{dim}_{\mathbb{F}_{q}}(M \cap T)=\operatorname{dim}_{\mathbb{F}_{q}} M=\operatorname{dim}_{\mathbb{F}_{q}} \bar{U}_{1}-\operatorname{dim}_{\mathbb{F}_{q}}\left(\bar{U}_{1} \cap V_{1}\right) \geq \\
\operatorname{dim}_{\mathbb{F}_{q}} \bar{U}_{1}-\operatorname{dim}_{\mathbb{F}_{q}}\left(U \cap V_{1}\right)=\operatorname{dim}_{\mathbb{F}_{q}} \bar{U}_{1}-\operatorname{dim}_{\mathbb{F}_{q}} U_{1},
\end{gathered}
$$

and hence, by (8),

$$
\operatorname{dim}_{\mathbb{F}_{q}}(M \cap T) \geq h-s+1,
$$

a contradiction.

The last part follows from $r n /(h+1)=\sum_{i=1}^{t} r_{i} n /(h+1)$.

Constructions of maximum 1-scattered $\mathbb{F}_{q}$-subspaces of $V\left(r, q^{n}\right)$ exist for all values of $q, r$ and $n$, provided $r n$ is even [1, 3, 4, 11]. For $r=3, n \leq 5$ see [2, Section 5]. Also, there are constructions of maximum $(r-1)$-scattered $\mathbb{F}_{q}$-subspaces arising from MRD-codes (explained later in Section 4.1) for all values of $q, r$ and $n$, cf. [28, Corollary 4.4]. In particular, the so called Gabidulin codes produce Example 2.5. One can also prove directly that these are maximum $(r-1)$-scattered subspaces by the same arguments as in the proof of Lemma 2.2 .

Example 2.5. In $\mathbb{F}_{q^{n}}^{r}$, if $n \geq r$, then the $\mathbb{F}_{q^{-}}$subspace

$$
\left\{\left(x, x^{q}, x^{q^{2}}, \ldots, x^{q^{r-1}}\right): x \in \mathbb{F}_{q^{n}}\right\}
$$

is maximum $(r-1)$-scattered of dimension $n$.

Theorem 2.6. If $h+1$ divides $r$ and $n \geq h+1$, then in $V=V\left(r, q^{n}\right)$ there exist maximum $h$-scattered $\mathbb{F}_{q}$-subspaces of dimension $r n /(h+1)$. 
Proof. Put $r=t(h+1)$ and consider $V=V_{1} \oplus \ldots \oplus V_{t}$, with $V_{i}$ an $\mathbb{F}_{q^{n-}}$ subspace of $V$ with dimension $h+1$. For each $i$ consider a maximum $h$ scattered $\mathbb{F}_{q}$-subspace $U_{i}$ in $V_{i}$ of dimension $n$ which exists because of Example 2.5. By Theorem 2.4, $U_{1} \oplus \ldots \oplus U_{t}$ is an $h$-scattered $\mathbb{F}_{q}$-subspace of $V$ with dimension $t n=\frac{r n}{h+1}$.

In Theorem 2.6 we exhibit examples of maximum $h$-scattered subspaces of $V=V\left(r, q^{n}\right)$ whenever $h+1$ divides $r$. In Section 3 we introduce a method to construct such subspaces also when $h+1$ does not divide $r$. To do this, we will need an upper bound on the dimension of intersections of hyperplanes of $V$ with a maximum $h$-scattered subspace of dimension $r n /(h+1)$. The proof of the following theorem is developed in Section 5 .

Theorem 2.7. If $U$ is a maximum $h$-scattered $\mathbb{F}_{q}$-subspace of a vector space $V\left(r, q^{n}\right)$ of dimension $r n /(h+1)$, then for any $(r-1)$-dimensional $\mathbb{F}_{q^{n}}$ subspace $W$ of $V\left(r, q^{n}\right)$ we have

$$
\frac{r n}{(h+1)}-n \leq \operatorname{dim}_{\mathbb{F}_{q}}(U \cap W) \leq \frac{r n}{(h+1)}-n+h .
$$

The above theorem is a generalisation of [4, Theorem 4.2] and the first part of its proof relies on the counting technique developed in [4, Theorem 4.2].

\section{Delsarte dual of an $h$-scattered subspace}

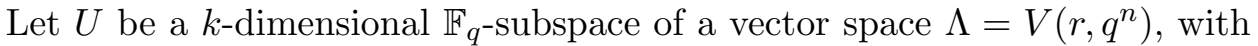
$k>r$. By [21, Theorems 1, 2] (see also [20, Theorem 1]), there is an embedding of $\Lambda$ in $\mathbb{V}=V\left(k, q^{n}\right)$ with $\mathbb{V}=\Lambda \oplus \Gamma$ for some $(k-r)$-dimensional

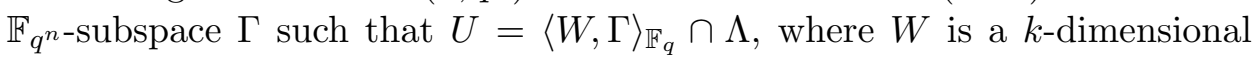

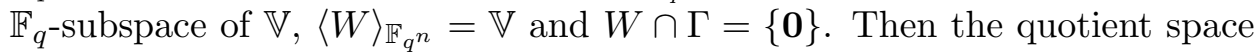
$\mathbb{V} / \Gamma$ is isomorphic to $\Lambda$ and under this isomorphism $U$ is the image of the

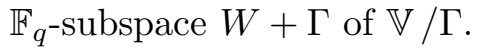

Now, let $\beta^{\prime}: W \times W \rightarrow \mathbb{F}_{q}$ be a non-degenerate reflexive sesquilinear form on $W$ with companion automorphism $\sigma^{\prime}$. Then $\beta^{\prime}$ can be extended to a non-degenerate reflexive sesquilinear form $\beta: \mathbb{V} \times \mathbb{V} \rightarrow \mathbb{F}_{q^{n}}$. Indeed if

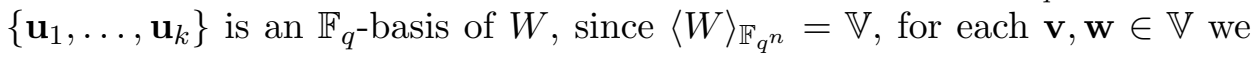
have

$$
\beta(\mathbf{v}, \mathbf{w})=\sum_{i, j=1}^{k} a_{i} b_{j}^{\sigma} \beta^{\prime}\left(\mathbf{u}_{i}, \mathbf{u}_{j}\right),
$$


where $\mathbf{v}=\sum_{i=1}^{k} a_{i} \mathbf{u}_{i}, \mathbf{w}=\sum_{i=1}^{k} b_{i} \mathbf{u}_{i}$ and $\sigma$ is an automorphism of $\mathbb{F}_{q^{n}}$ such that $\sigma_{\mid \mathbb{F}_{q}}=\sigma^{\prime}$. Let $\perp$ and $\perp^{\prime}$ be the orthogonal complement maps defined

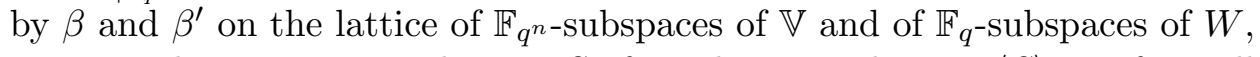

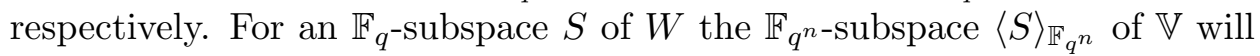
be denoted by $S^{*}$. In this case $\left(S^{*}\right)^{\perp}=\left(S^{\perp^{\prime}}\right)^{*}$.

In this setting, we can prove the following preliminary result.

Proposition 3.1. Let $W, \Lambda, \Gamma, \mathbb{V}, \perp$ and $\perp^{\prime}$ be defined as above. If $U$ is a $k$-dimensional $\mathbb{F}_{q}$-subspace of $\Lambda$ with $k>r$ and

$$
\operatorname{dim}_{\mathbb{F}_{q}}(M \cap U)<k-1 \text { holds for each hyperplane } M \text { of } \Lambda,
$$

then $W+\Gamma^{\perp}$ is a $k$-dimensional $\mathbb{F}_{q^{-}}$-subspace of the quotient space $\mathbb{V} / \Gamma^{\perp}$.

Proof. As described above, $U$ turns out to be isomorphic to the $\mathbb{F}_{q^{-}}$-subspace $W+\Gamma$ of the quotient space $\mathbb{V} / \Gamma$. By $(\diamond)$, since each hyperplane of $\mathbb{V} / \Gamma$ is of form $H+\Gamma$ where $H$ is a hyperplane of $\mathbb{V}$ containing $\Gamma$, it follows that

$$
\operatorname{dim}_{\mathbb{F}_{q}}(H \cap W)<k-1 \text { for each hyperplane } H \text { of } \mathbb{V} \text { containing } \Gamma \text {. }
$$

To prove the assertion it is enough to prove

$$
W \cap \Gamma^{\perp}=\{\mathbf{0}\} .
$$

Indeed, by way of contradiction, suppose that there exists a nonzero vector

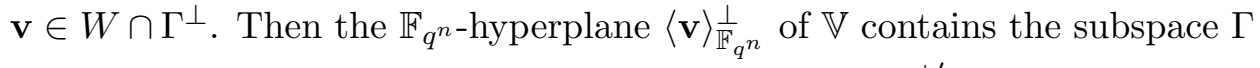
and meets $W$ in the $(k-1)$-dimensional $\mathbb{F}_{q^{-}}$-subspace $\langle\mathbf{v}\rangle_{\mathbb{F}_{q}^{\prime}}$, which contradicts $\diamond \infty$.

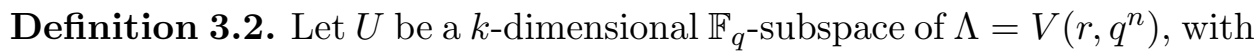
$k>r$ and such that $\otimes$ is satisfied. Then the $k$-dimensional $\mathbb{F}_{q^{-} \text {-subspace }}$ $W+\Gamma^{\perp}$ of the quotient space $\mathbb{V} / \Gamma^{\perp}$ (cf. Proposition 3.1) will be denoted by $\bar{U}$ and we call it the Delsarte dual of $U$ (w.r.t. $\perp$ ).

The term Delsarte dual comes from the Delsarte dual operation acting on MRD-codes, as pointed out in Theorem 4.12 .

Theorem 3.3. Let $U$ be a maximum $h$-scattered $\mathbb{F}_{q}$-subspace of a vector space $\Lambda=V\left(r, q^{n}\right)$ of dimension $r n /(h+1)$, with $n \geq h+3$. Then the

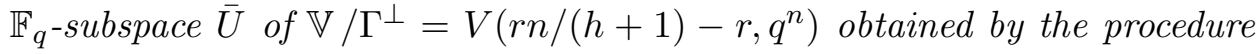
of Proposition 3.1 is maximum $(n-h-2)$-scattered. 
Proof. Put $k:=r n /(h+1)$. We first note that condition $(\otimes)$ is satisfied for $U$ since by Theorem 2.7 the hyperplanes of $\Lambda$ meet $U$ in $\mathbb{F}_{q}$-subspaces of dimension at most $r n /(h+1)-n+h<k-1$. Also, $k>r$ holds since $n \geq h+3$.

Hence we can apply the procedure of Proposition 3.1 to obtain the $\mathbb{F}_{q^{-}}$ subspace $\bar{U}=W+\Gamma^{\perp}$ of $\mathbb{V} / \Gamma^{\perp}$ of dimension $k$.

By way of contradiction, suppose that there exists an $(n-h-2)$ -

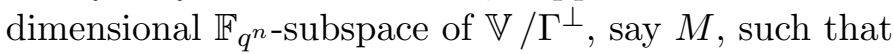

$$
\operatorname{dim}_{\mathbb{F}_{q}}(M \cap \bar{U}) \geq n-h-1 .
$$

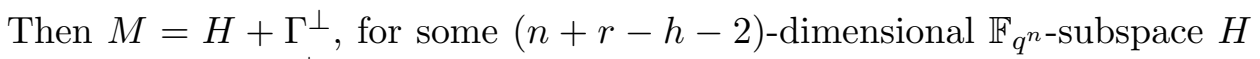
of $\mathbb{V}$ containing $\Gamma^{\perp}$. For $H$, by (9), it follows that

$$
\operatorname{dim}_{\mathbb{F}_{q}}(H \cap W)=\operatorname{dim}_{\mathbb{F}_{q}}(M \cap \bar{U}) \geq n-h-1 .
$$

Let $S$ be an $(n-h-1)$-dimensional $\mathbb{F}_{q^{-}}$-subspace of $W$ contained in $H$ and let $S^{*}:=\langle S\rangle_{\mathbb{F}_{q^{n}}}$. Then, $\operatorname{dim}_{\mathbb{F}_{q^{n}}} S^{*}=n-h-1$,

$$
S^{\perp^{\prime}}=W \cap\left(S^{*}\right)^{\perp} \quad \text { and } \quad S^{\perp^{\prime}} \subset\left(S^{*}\right)^{\perp}=\left\langle S^{\perp^{\prime}}\right\rangle_{\mathbb{F}_{q^{n}}} .
$$

Since $S \subseteq H \cap W$ and $\Gamma^{\perp} \subset H$, we get $S^{*} \subset H$ and $H^{\perp} \subset \Gamma$, i.e.

$$
H^{\perp} \subseteq \Gamma \cap\left(S^{*}\right)^{\perp} .
$$

From (11) it follows that

$$
\operatorname{dim}_{\mathbb{F}_{q^{n}}}\left(\Gamma \cap\left(S^{*}\right)^{\perp}\right) \geq \operatorname{dim}_{\mathbb{F}_{q^{n}}} H^{\perp}=k-(n+r-h-2) .
$$

This implies that

$$
\operatorname{dim}_{\mathbb{F}_{q^{n}}}\left\langle\Gamma,\left(S^{*}\right)^{\perp}\right\rangle_{\mathbb{F}_{q^{n}}}=\operatorname{dim}_{\mathbb{F}_{q^{n}}} \Gamma+\operatorname{dim}_{\mathbb{F}_{q^{n}}}\left(S^{*}\right)^{\perp}-\operatorname{dim}_{\mathbb{F}_{q^{n}}}\left(\Gamma \cap\left(S^{*}\right)^{\perp}\right) \leq k-1
$$

and hence $\left\langle\Gamma,\left(S^{*}\right)^{\perp}\right\rangle_{\mathbb{F}_{q^{n}}}$ is contained in a hyperplane $T$ of $\mathbb{V}$ containing $\Gamma$. Also, $\operatorname{dim}_{\mathbb{F}_{q}}\left(S^{\perp^{\prime}}\right)=\operatorname{dim}_{\mathbb{F}_{q}} W-\operatorname{dim}_{\mathbb{F}_{q}} S=k-(n-h-1)$ and, by (10), we get

$$
S^{\perp^{\prime}}=W \cap\left(S^{*}\right)^{\perp} \subseteq W \cap T .
$$

Then $\hat{T}:=T \cap \Lambda$ is a hyperplane of $\Lambda$ and, by recalling $U=\langle W, \Gamma\rangle_{\mathbb{F}_{q}} \cap \Lambda$,

$$
\operatorname{dim}_{\mathbb{F}_{q}}(\hat{T} \cap U)=\operatorname{dim}_{\mathbb{F}_{q}}(T \cap W) \geq \operatorname{dim}_{\mathbb{F}_{q}}\left(S^{\perp^{\prime}}\right)=k-n+h+1,
$$

contradicting Theorem 2.7 . 
In case of $h=r-1$, Theorem 3.3 follows from [28] and from the theory of MRD codes. Our theorem generalises this result to each value of $h$ by using a geometric approach.

Corollary 3.4. Starting from a maximum $(r-1)$-scattered $\mathbb{F}_{q}$-subspace $U$ of $V\left(r, q^{n}\right)$ of dimension $n, n \geq r+2$, the $\mathbb{F}_{q}$-subspace $\bar{U}$ (cf. Definition 3.2) is a maximum $(n-r-1)$-scattered $\mathbb{F}_{q}$-subspace of $V\left(n-r, q^{n}\right)$ of dimension $n$.

Corollary 3.5. Starting from a maximum 1-scattered $\mathbb{F}_{q}$-subspace $U$ of $V\left(r, q^{n}\right)$, rn even, $n \geq 4, \bar{U}$ (cf. Definition 3.2) is a maximum $(n-3)$ scattered $\mathbb{F}_{q}$-subspace of $V\left(r(n-2) / 2, q^{n}\right)$ whose dimension attains bound (1).

Theorem 3.6. If $n \geq 4$ is even and $r \geq 3$ is odd, then there exist maximum

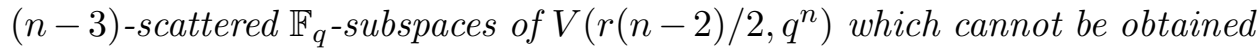
from the direct sum construction of Theorem 2.6.

Proof. By [1, 3, 4, 11] it is always possible to construct maximum 1-scattered

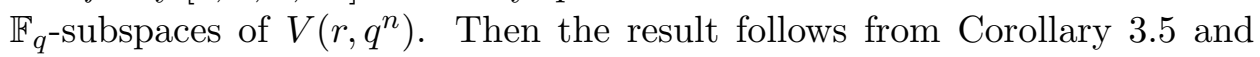
from the fact that in this case $n-2$ does not divide $r(n-2) / 2$.

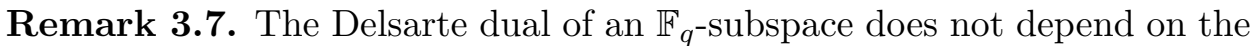
choice of the non-degenerate reflexive sesquilinear form on $W$.

Indeed, fix an $\mathbb{F}_{q^{-}}$basis $B$ of $W$, since $\langle W\rangle_{\mathbb{F}_{q^{n}}}=\mathbb{V}$, we can see $W$ as $\mathbb{F}_{q}^{k}$ and $\mathbb{V}$ as $\mathbb{F}_{q^{n}}^{k}$. Let $\beta_{1}^{\prime}$ and $\beta_{2}^{\prime}$ be two non-degenerate reflexive sesquilinear forms on $\mathbb{F}_{q}^{k}$. Then, with respect to the basis $B$, the forms $\beta_{1}^{\prime}$ and $\beta_{2}^{\prime}$ are defined by the following rules:

$$
\beta_{i}^{\prime}((\mathbf{x}, \mathbf{y}))=\mathbf{x} G_{i} \mathbf{y}_{t}^{\rho_{i}} \square
$$

where $G_{i} \in \operatorname{GL}(k, q)$ and $\rho_{i}$ is an automorphism of $\mathbb{F}_{q}$ such that $\rho_{i}^{2}=\mathrm{id}$ and $\left(G_{i}^{\rho_{i}}\right)^{t}=G_{i}$, for $i \in\{1,2\}$. Now let $\beta_{1}$ and $\beta_{2}$ be their extensions over $\mathbb{F}_{q^{n}}^{k}$ defined by the rules

$$
\beta_{i}((\mathbf{x}, \mathbf{y}))=\mathbf{x} G_{i} \mathbf{y}_{t}^{\rho_{i}}
$$

and let $\perp_{1}$ and $\perp_{2}$ be the orthogonal complement maps defined by $\beta_{1}$ and

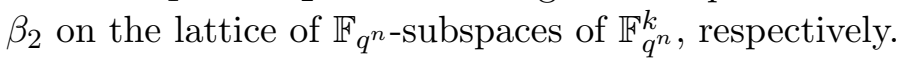

Again w.r.t. the basis $B$, the $\mathbb{F}_{q^{n}}$-subspace $\Gamma$ described at the beginning of this section can be seen as a $(k-r)$-dimensional subspace of $\mathbb{F}_{q^{n}}^{k}$. Then, for $i \in\{1,2\}$ we have

$$
\Gamma^{\perp_{i}}=\left\{\mathbf{x}: \mathbf{x} G_{i} \mathbf{y}_{t}^{\rho_{i}}=0 \quad \forall \mathbf{y} \in \Gamma\right\} .
$$

\footnotetext{
${ }^{1}$ Here $\mathbf{y}_{t}$ denotes the transpose of the vector $\mathbf{y}$.
} 
Straightforward computations show that the invertible semilinear map

$$
\varphi: \mathbf{x} \in \mathbb{F}_{q^{n}}^{k} \mapsto \mathbf{x}^{\rho_{2}^{-1} \rho_{1}} G_{2}^{\rho_{2}^{-1} \rho_{1}} G_{1}^{-1} \in \mathbb{F}_{q^{n}}^{k}
$$

leaves $W$ invariant and maps $\Gamma^{\perp_{2}}$ to $\Gamma^{\perp_{1}}$. Then $\varphi$ maps $W+\Gamma^{\perp_{2}}$ to $W+\Gamma^{\perp_{1}}$, i.e. $\varphi$ maps the Delsarte dual of $U$ calculated w.r.t $\beta_{2}$ to the Delsarte dual of $U$ calculated w.r.t. $\beta_{1}$. See also [25, Section 2] and [27, Section 6.2].

\section{Linear sets defined by $h$-scattered subspaces}

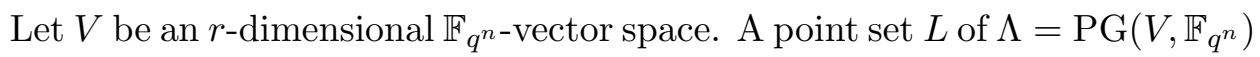
$=\mathrm{PG}\left(r-1, q^{n}\right)$ is said to be an $\mathbb{F}_{q}$-linear set of $\Lambda$ of rank $k$ if it is defined by the non-zero vectors of a $k$-dimensional $\mathbb{F}_{q}$-vector subspace $U$ of $V$, i.e.

$$
L=L_{U}:=\left\{\langle\mathbf{u}\rangle_{\mathbb{F}_{q^{n}}}: \mathbf{u} \in U \backslash\{\mathbf{0}\}\right\}
$$

One of the most natural questions about linear sets is their equivalence. Two linear sets $L_{U}$ and $L_{W}$ of $\mathrm{PG}\left(r-1, q^{n}\right)$ are said to be PГL-equivalent (or simply equivalent) if there is an element $\varphi$ in $\operatorname{P\Gamma L}\left(r, q^{n}\right)$ such that $L_{U}^{\varphi}=L_{W}$. In the applications it is crucial to have methods to decide whether two linear sets are equivalent or not. This can be a difficult problem and some results in this direction can be found in [9, 8, 12]. For $f \in \Gamma L\left(r, q^{n}\right)$ we have $L_{U^{f}}=L_{U}^{\varphi_{f}}$, where $\varphi_{f}$ denotes the collineation of $\operatorname{PG}\left(V, \mathbb{F}_{q^{n}}\right)$ induced by $f$. It follows that if $U$ and $W$ are $\mathbb{F}_{q}$-subspaces of $V$ belonging to the same orbit of $\Gamma \mathrm{L}\left(r, q^{n}\right)$, then $L_{U}$ and $L_{W}$ are equivalent. The above condition is only sufficient but not necessary to obtain equivalent linear sets. This

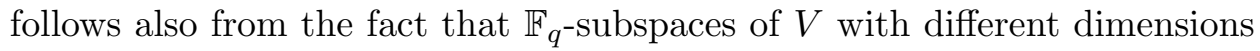
can define the same linear set, for example $\mathbb{F}_{q^{-}}$linear sets of $\operatorname{PG}\left(r-1, q^{n}\right)$ of rank $k \geq r n-n+1$ are all the same: they coincide with $\operatorname{PG}\left(r-1, q^{n}\right)$. Also, in [8, 12] for $r=2$ it was pointed out that there exist maximum

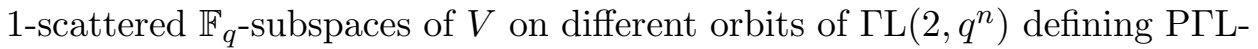
equivalent linear sets of $\mathrm{PG}\left(1, q^{n}\right)$. It is then natural to ask for which linear sets can we translate the question of PCL-equivalence into the question of $\Gamma$-equivalence of the defining subspaces. For further details on linear sets see [17, 18, 24].

In this section we study the equivalence issue of $\mathbb{F}_{q}$-linear sets defined by $h$-scattered linear sets for $h \geq 2$.

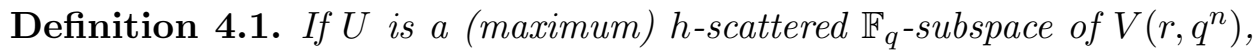
then the $\mathbb{F}_{q}$-linear set $L_{U}$ of $\mathrm{PG}\left(r-1, q^{n}\right)$ is called (maximum) h-scattered. 
The $(r-1)$-scattered $\mathbb{F}_{q}$-linear sets of rank $n$ were defined also in [28, Definition 14] and following the authors of [28], we will call these $\mathbb{F}_{q}$-linear sets maximum scattered with respect to hyperplanes. Also, we will call 2scattered $\mathbb{F}_{q}$-linear sets (of any rank) scattered with respect to lines.

Proposition 4.2 ([5, pg. 3 Eq. (6) and Lemma 2.1]). Let $V$ be a twodimensional vector space over $\mathbb{F}_{q^{n}}$.

1. If $U$ is an $\mathbb{F}_{q}$-subspace of $V$ with $\left|L_{U}\right|=q+1$, then $U$ has dimension 2 over $\mathbb{F}_{q}$.

2. Let $U$ and $W$ be two $\mathbb{F}_{q}$-subspaces of $V$ with $L_{U}=L_{W}$ of size $q+1$. If $U \cap W \neq\{\mathbf{0}\}$, then $U=W$.

Proposition 4.3. If $L_{U}$ is a scattered $\mathbb{F}_{q}$-linear set with respect to lines of $\mathrm{PG}\left(r-1, q^{n}\right)=\mathrm{PG}\left(V, \mathbb{F}_{q^{n}}\right)$, then its rank is uniquely defined, i.e. for each $\mathbb{F}_{q}$-subspace $W$ of $V$ if $L_{W}=L_{U}$, then $\operatorname{dim}_{\mathbb{F}_{q}} W=\operatorname{dim}_{\mathbb{F}_{q}} U$.

Proof. Let $W$ be an $\mathbb{F}_{q}$-subspace of $V$ such that $L_{U}=L_{W}$ and put $k=$

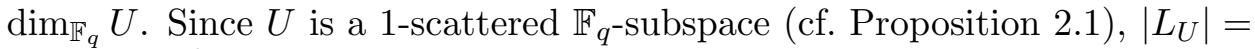
$\left|L_{W}\right|=\left(q^{k}-1\right) /(q-1)$. It follows that $\operatorname{dim}_{\mathbb{F}_{q}} W \geq k$. Suppose that $\operatorname{dim}_{\mathbb{F}_{q}} W \geq k+1$, then there exists at least one point $P=\langle\mathbf{x}\rangle_{\mathbb{F}_{q^{n}}} \in L_{W}$ such that $\operatorname{dim}_{\mathbb{F}_{q}}\left(W \cap\langle\mathbf{x}\rangle_{\mathbb{F}_{q^{n}}}\right) \geq 2$. Let $Q=\langle\mathbf{y}\rangle_{\mathbb{F}_{q^{n}}} \in L_{U}=L_{W}$ be a point different from $P$, then $\langle\mathbf{x}, \mathbf{y}\rangle_{\mathbb{F}_{q^{n}}} \cap W$ has dimension at least 3 but the linear set defined by $\langle\mathbf{x}, \mathbf{y}\rangle_{\mathbb{F}_{q^{n}}} \cap W$ is $L_{W} \cap\langle P, Q\rangle$, thus it has size $q+1$, contradicting part 1 of Proposition 4.2 .

Lemma 4.4. Let $L_{U}$ be a scattered $\mathbb{F}_{q}$-linear set with respect to lines in $\operatorname{PG}\left(r-1, q^{n}\right)$. If $L_{U}=L_{W}$ for some $\mathbb{F}_{q}$-subspace $W$, then $U=\lambda W$ for some $\lambda \in \mathbb{F}_{q^{n}}^{*}$.

Proof. By Proposition 4.3, we have $\operatorname{dim}_{\mathbb{F}_{q}} W=\operatorname{dim}_{\mathbb{F}_{q}} U$ and hence, since $U$ is 1-scattered, also $W$ is 1-scattered. Let $P \in L_{U}$ with $P=\langle\mathbf{u}\rangle_{\mathbb{F}_{q^{n}}}$, then for some $\lambda \in \mathbb{F}_{q^{n}}^{*}$ we have $\mathbf{u} \in U \cap \lambda W$. Put $W^{\prime}:=\lambda W$ and note that $L_{W}=L_{W^{\prime}}$. Our aim is to prove $W^{\prime} \subseteq U$. Since $U$ and $W^{\prime}$ are 1-scattered, we have $\langle\mathbf{u}\rangle_{\mathbb{F}_{q^{n}}} \cap U=\langle\mathbf{u}\rangle_{\mathbb{F}_{q^{n}}} \cap W^{\prime}=\langle\mathbf{u}\rangle_{\mathbb{F}_{q}}$.

What is left, is to show for each $\mathbf{w} \in W^{\prime} \backslash\langle\mathbf{u}\rangle_{\mathbb{F}_{q^{n}}}$ that $\mathbf{w} \in U$. To do this, consider the point $Q=\langle\mathbf{w}\rangle_{\mathbb{F}_{q^{n}}} \in L_{W^{\prime}}=L_{U}$ and the line $\langle P, Q\rangle$ which meets $L_{U}$ in $q+1$ points. By part 1 of Proposition 4.2, the $\mathbb{F}_{q}$-subspace $\left(\langle\mathbf{u}, \mathbf{w}\rangle_{\mathbb{F}_{q^{n}}} \cap U\right)$ has dimension 2. Since $\left(\langle\mathbf{u}, \mathbf{w}\rangle_{\mathbb{F}_{q^{n}}} \cap U\right) \cap\left(\langle\mathbf{u}, \mathbf{w}\rangle_{\mathbb{F}_{q^{n}}} \cap W^{\prime}\right) \neq$ $\{\mathbf{0}\}$, by part 2 of Proposition 4.2 we get

$$
\langle\mathbf{u}, \mathbf{w}\rangle_{\mathbb{F}_{q^{n}}} \cap U=\langle\mathbf{u}, \mathbf{w}\rangle_{\mathbb{F}_{q^{n}}} \cap W^{\prime}=\langle\mathbf{u}, \mathbf{w}\rangle_{\mathbb{F}_{q}} .
$$

Hence the assertion follows. 
Theorem 4.5. Consider two h-scattered linear sets $L_{U}$ and $L_{W}$ of $V\left(r, q^{n}\right)$ with $h \geq 2$. They are $\operatorname{P\Gamma L}\left(r, q^{n}\right)$-equivalent if and only if $U$ and $W$ are $\Gamma \mathrm{L}\left(r, q^{n}\right)$-equivalent.

Proof. The if part is trivial. To prove the only if part assume that there exists $f \in \Gamma L\left(r, q^{n}\right)$ such that $L_{U}^{\varphi_{f}}=L_{W}$, where $\varphi_{f}$ is the collineation induced by $f$. Since $L_{U}^{\varphi_{f}}=L_{U^{f}}$, by Proposition 2.1 and Lemma 4.4 , there exists $\lambda \in \mathbb{F}_{q^{n}}^{*}$ such that $\lambda U^{f}=W$ and hence $U$ and $W$ lie on the same orbit of $\Gamma \mathrm{L}\left(r, q^{n}\right)$.

\subsection{Scattered linear sets with respect to hyperplanes and MRD-codes}

A rank distance (or $R D$ ) code $\mathcal{C}$ of $\mathbb{F}_{q}^{n \times m}, n \leq m$, can be considered as a subset of $\operatorname{Hom}_{\mathbb{F}_{q}}(U, V)$, where $\operatorname{dim}_{\mathbb{F}_{q}} U=m$ and $\operatorname{dim}_{\mathbb{F}_{q}} V=n$, with rank distance defined as $d(f, g):=\operatorname{rk}(f-g)$. The minimum distance of $\mathcal{C}$ is $d:=\min \{d(f, g): f, g \in \mathcal{C}, f \neq g\}$.

Result 4.6 ([13]). If $\mathcal{C}$ is a rank distance code of $\mathbb{F}_{q}^{n \times m}, n \leq m$, with minimum distance $d$, then

$$
|\mathcal{C}| \leq q^{m(n-d+1)} .
$$

Rank distance codes for which $(12)$ holds with equality are called maximum rank distance (or MRD) codes.

From now on, we will only consider $\mathbb{F}_{q^{-}}$-linear MRD-codes of $\mathbb{F}_{q}^{n \times n}$, i.e.

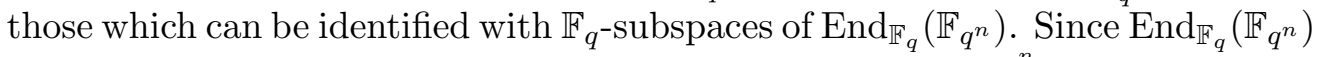
is isomorphic to the ring of $q$-polynomials over $\mathbb{F}_{q^{n}}$ modulo $x^{q^{n}}-x$, denoted by $\mathcal{L}_{n, q}$, with addition and composition as operations, we will consider $\mathcal{C}$ as an $\mathbb{F}_{q}$-subspace of $\mathcal{L}_{n, q}$. Given two $\mathbb{F}_{q}$-linear MRD codes, $\mathcal{C}_{1}$ and $\mathcal{C}_{2}$, they are equivalent if and only if there exist $\varphi_{1}, \varphi_{2} \in \mathcal{L}_{n, q}$ permuting $\mathbb{F}_{q^{n}}$ and $\rho \in \operatorname{Aut}\left(\mathbb{F}_{q}\right)$ such that

$$
\varphi_{1} \circ f^{\rho} \circ \varphi_{2} \in \mathcal{C}_{2} \text { for all } f \in \mathcal{C}_{1},
$$

where $\circ$ stands for the composition of maps and $f^{\rho}(x)=\sum a_{i}^{\rho} x^{q^{i}}$ for $f(x)=$ $\sum a_{i} x^{q^{i}}$. For a rank distance code $\mathcal{C}$ given by a set of linearized polynomials, its left and right idealisers can be written as:

$$
\begin{aligned}
& L(\mathcal{C})=\left\{\varphi \in \mathcal{L}_{n, q}: \varphi \circ f \in \mathcal{C} \text { for all } f \in \mathcal{C}\right\}, \\
& R(\mathcal{C})=\left\{\varphi \in \mathcal{L}_{n, q}: f \circ \varphi \in \mathcal{C} \text { for all } f \in \mathcal{C}\right\} .
\end{aligned}
$$

By [19, Section 2.7] and [28] the next result follows. We give a proof of the first part for the sake of completeness. 
Result 4.7. $\mathcal{C}$ is an $\mathbb{F}_{q}$-linear $M R D$-code of $\mathcal{L}_{n, q}$ with minimum distance $n-r+1$ and with left-idealiser isomorphic to $\mathbb{F}_{q^{n}}$ if and only if up to equivalence

$$
\mathcal{C}=\left\langle f_{1}(x), \ldots, f_{r}(x)\right\rangle_{\mathbb{F}_{q^{n}}}
$$

for some $f_{1}, f_{2}, \ldots, f_{r} \in \mathcal{L}_{n, q}$ and the $\mathbb{F}_{q}$-subspace

$$
U_{\mathcal{C}}=\left\{\left(f_{1}(x), \ldots, f_{r}(x)\right): x \in \mathbb{F}_{q^{n}}\right\}
$$

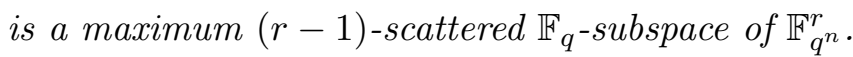

Proof. Let $T=\left\{\omega_{a}: a \in \mathbb{F}_{q^{n}}\right\}$, where for each $a \in \mathbb{F}_{q^{n}}, \omega_{a}(x)=a x \in \mathcal{L}_{n, q}$ and let $L$ denote the left-idealiser of $\mathcal{C}$. Since $T$ and $L$ are Singer cyclic subgroups of $\mathrm{GL}\left(\mathbb{F}_{q^{n}}, \mathbb{F}_{q}\right)$ and any two such groups are conjugate (cf. [16, pg. 187]) it follows that there exists an invertible $q$-polynomial $g$ such that $g \circ L \circ g^{-1}=T$. Then for each $h \in \mathcal{C}^{\prime}:=g^{-1} \circ \mathcal{C}$ it holds that $\omega_{a} \circ h \in \mathcal{C}^{\prime}$ for each $a \in \mathbb{F}_{q^{n}}$, which proves the first statement. For the second part see [28, Corollary 4.4].

Remark 4.8. The adjoint of a q-polynomial $f(x)=\sum_{i=0}^{n-1} a_{i} x^{q^{i}}$, with respect to the bilinear form $\left.\langle x, y\rangle:=\operatorname{Tr}_{q^{n} / q}(x y){ }^{2}\right)$, is given by

$$
\hat{f}(x):=\sum_{i=0}^{n-1} a_{i}^{q^{n-i}} x^{q^{n-i}} .
$$

If $\mathcal{C}$ is a rank distance code given by q-polynomials, then the adjoint code $\mathcal{C}^{\top}$ of $\mathcal{C}$ is $\{\hat{f}: f \in \mathcal{C}\}$. The code $\mathcal{C}$ is an $M R D$ if and only if $\mathcal{C}^{\top}$ is an $M R D$ and also $L(\mathcal{C}) \cong R\left(\mathcal{C}^{\top}\right), R(\mathcal{C}) \cong L\left(\mathcal{C}^{\top}\right)$. Thus Result 4.7 can be translated also to codes with right-idealiser isomorphic to $\mathbb{F}_{q^{n}}$.

The next result follows from [28, Proposition 3.5].

Result 4.9. Let $\mathcal{C}$ and $\mathcal{C}^{\prime}$ be two $\mathbb{F}_{q}$-linear $M R D$-codes of $\mathcal{L}_{n, q}$ with minimum distance $n-r+1$ and with left-idealisers isomorphic to $\mathbb{F}_{q^{n}}$. Then $U_{\mathcal{C}}$ and $U_{\mathcal{C}^{\prime}}$ are $\Gamma \mathrm{L}\left(r, q^{n}\right)$-equivalent if and only if $\mathcal{C}$ and $\mathcal{C}^{\prime}$ are equivalent.

By Theorem 4.5, for $r>2$ we can extend Result 4.9 in the following way.

Theorem 4.10. Let $\mathcal{C}$ and $\mathcal{C}^{\prime}$ be two $\mathbb{F}_{q}$-linear $M R D$-codes of $\mathcal{L}_{n, q}$ with minimum distance $n-r+1, r>2$, and with left-idealisers isomorphic to $\mathbb{F}_{q^{n}}$. Then the linear sets $L_{U_{\mathcal{C}}}$ and $L_{U_{\mathcal{C}^{\prime}}}$ are $\operatorname{P\Gamma L}\left(r, q^{n}\right)$-equivalent if and only if $\mathcal{C}$ and $\mathcal{C}^{\prime}$ are equivalent.

${ }^{2}$ Where $\operatorname{Tr}_{q^{n} / q}(x)=x+x^{q}+\ldots+x^{q^{n-1}}$ denotes the $\mathbb{F}_{q^{n}} \rightarrow \mathbb{F}_{q}$ trace function. 
In the following we motivate why we used the term "Delsarte dual" in Definition 3.2. In particular, we prove that the duality of Section 3 corresponds to the Delsarte duality on MRD-codes when $(r-1)$-scattered

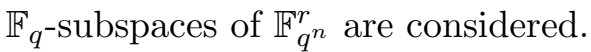

First recall that in terms of linearized polynomials, the Delsarte dual of a rank distance code $\mathcal{C}$ of $\mathcal{L}_{n, q}$ introduced in [13] and in [14] can be interpreted as follows

$$
\mathcal{C}^{\perp}=\left\{f \in \mathcal{L}_{n, q}: b(f, g)=0 \forall g \in \mathcal{C}\right\},
$$

where $b(f, g)=\operatorname{Tr}_{q^{n} / q}\left(\sum_{i=0}^{n-1} a_{i} b_{i}\right)$ for $f(x)=\sum_{i=0}^{n-1} a_{i} x^{q^{i}}$ and $g(x)=$ $\sum_{i=0}^{n-1} b_{i} x^{q^{i}} \in \mathcal{L}_{n, q}$.

Remark 4.11. Let $\mathcal{C}$ be an $\mathbb{F}_{q}$-linear MRD-code of $\mathcal{L}_{n, q}$ with minimum distance $n-r+1$ and with left-idealiser isomorphic to $\mathbb{F}_{q^{n}}$. By Result 4.7 and by [10, Theorem 2.2], there exist $r$ distinct integers in $\{0, \ldots, n-1\}$ such that, up to equivalence,

$$
\mathcal{C}=\left\langle h_{0}(x), \ldots, h_{r-1}(x)\right\rangle_{\mathbb{F}_{q^{n}}}
$$

where

$$
h_{i}(x)=x^{q^{t_{i}}}+\sum_{j \notin\left\{t_{0}, \ldots, t_{r-1}\right\}} g_{i, j} x^{q^{j}}
$$

and $g_{i, j} \in \mathbb{F}_{q^{n}}$.

Also, let $\left\{s_{0}, s_{1}, \ldots, s_{n-r-1}\right\}:=\{0, \ldots, n-1\} \backslash\left\{t_{0}, \ldots, t_{r-1}\right\}$. Then it is easy to see that the Delsarte dual of $\mathcal{C}$ is

$$
\mathcal{C}^{\perp}=\left\langle h_{0}^{\prime}(x), \ldots, h_{n-r-1}^{\prime}(x)\right\rangle_{\mathbb{F}_{q} n},
$$

where

$$
h_{i}^{\prime}(x)=x^{q^{s_{i}}}-\sum_{j \in\left\{t_{0}, \ldots, t_{r-1}\right\}} g_{j, s_{i}} x^{q^{j}} .
$$

Theorem 4.12. Let $\mathcal{C}$ be an $\mathbb{F}_{q}$-linear $M R D$-code of $\mathcal{L}_{n, q}$ with minimum distance $n-r+1$ and with left-idealiser isomorphic to $\mathbb{F}_{q^{n}}$. Then there exist $h_{0}(x), \ldots, h_{r-1}(x), h_{0}^{\prime}(x), \ldots, h_{n-r-1}^{\prime}(x) \in \mathcal{L}_{n, q}$ such that, up to equivalence,

- $\mathcal{C}=\left\langle h_{0}(x), \ldots, h_{r-1}(x)\right\rangle_{\mathbb{F}_{q^{n}}}$,

- $\mathcal{C}^{\perp}=\left\langle h_{0}^{\prime}(x), \ldots, h_{n-r-1}^{\prime}(x)\right\rangle_{\mathbb{F}_{q^{n}}}$,

- the Delsarte dual of $U_{\mathcal{C}}=\left\{\left(h_{0}(x), \ldots, h_{r-1}(x)\right): x \in \mathbb{F}_{q^{n}}\right\}$ is the $\mathbb{F}_{q^{-} \text {subspace }} U_{\mathcal{C}^{\perp}}=\left\{\left(h_{0}^{\prime}(x), \ldots, h_{n-r-1}^{\prime}(x)\right): x \in \mathbb{F}_{q^{n}}\right\}$. 
Proof. By Remark 4.11, up to equivalence, $\mathcal{C}=\left\langle h_{0}(x), \ldots, h_{r-1}(x)\right\rangle_{\mathbb{F}_{q^{n}}}$, for some $h_{0}(x), \ldots, h_{r-1}(x)$ as in $(13)$, and $\mathcal{C}^{\perp}=\left\langle h_{0}^{\prime}(x), \ldots, h_{n-r-1}^{\prime}(x)\right\rangle_{\mathbb{F}_{q^{n}}}$, for some $h_{0}^{\prime}(x), \ldots, h_{n-r-1}^{\prime}(x)$ as in (14). Note that, since $\mathcal{C}$ is an MRD-code, the linearized polynomials $h_{0}(x), \ldots, h_{r-1}(x)$ have no common roots other than 0 since otherwise the code would not contain invertible maps, see e.g. [22, Lemma 2.1]. Our aim is to show that applying the duality introduced in Section 3 to $U_{\mathcal{C}}=\left\{\left(h_{0}(x), \ldots, h_{r-1}(x)\right): x \in \mathbb{F}_{q^{n}}\right\}$ we get the $\mathbb{F}_{q^{-} \text {-subspace }}$ $U_{\mathcal{C}^{\perp}}=\left\{\left(h_{0}^{\prime}(x), \ldots, h_{n-r-1}^{\prime}(x)\right): x \in \mathbb{F}_{q^{n}}\right\}$. By Result 4.7 we have that $U_{\mathcal{C}}$

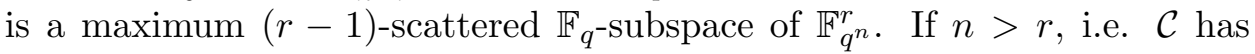
minimum distance greater than one, we can embed $\Lambda=\left\langle U_{\mathcal{C}}\right\rangle_{\mathbb{F}_{q^{n}}}$ in $\mathbb{F}_{q^{n}}^{n}$ in such a way that

$$
\Lambda=\left\{\left(x_{0}, x_{1}, \ldots, x_{r}, \ldots, x_{n-1}\right) \in \mathbb{F}_{q^{n}}^{n}: x_{j}=0 j \notin\left\{t_{0}, \ldots, t_{r-1}\right\}\right\},
$$

and hence the vector $\left(h_{0}(x), \ldots, h_{r-1}(x)\right)$ of $U_{\mathcal{C}}$ is extended to the vector $\left(a_{0}, a_{1}, \ldots, a_{n-1}\right)$ of $\mathbb{F}_{q^{n}}^{n}$ as follows

$$
a_{i}= \begin{cases}h_{i}(x) & \text { if } i \in\left\{t_{0}, \ldots, t_{r-1}\right\} \\ 0 & \text { otherwise }\end{cases}
$$

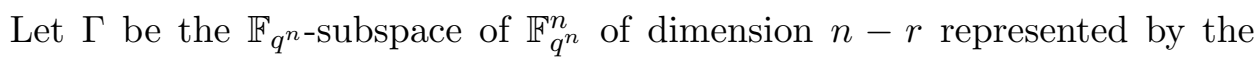
equations

$$
\Gamma:\left\{\begin{array}{l}
x_{t_{0}}=-\sum_{j \notin\left\{t_{0}, \ldots, t_{r-1}\right\}} g_{0, j} x_{j} \\
\vdots \\
x_{t_{r-1}}=-\sum_{j \notin\left\{t_{0}, \ldots, t_{r-1}\right\}} g_{r-1, j} x_{j}
\end{array}\right.
$$

and let $W=\left\{\left(x, x^{q}, \ldots, x^{q^{n-1}}\right): x \in \mathbb{F}_{q^{n}}\right\}$. It can be seen that $\Gamma \cap W=\{\mathbf{0}\}$, otherwise the polynomials $h_{0}(x), \ldots, h_{r-1}(x)$ would have a common root. Also

$$
U_{\mathcal{C}}=\langle W, \Gamma\rangle_{\mathbb{F}_{q}} \cap \Lambda \text {. }
$$

Let $\beta: \mathbb{F}_{q^{n}}^{n} \times \mathbb{F}_{q^{n}}^{n} \rightarrow \mathbb{F}_{q^{n}}$ be the standard inner product, i.e. $\beta((\mathbf{x}, \mathbf{y}))=$ $\sum_{i=0}^{n-1} x_{i} y_{i}$ where $\mathbf{x}=\left(x_{0}, \ldots, x_{n-1}\right)$ and $\mathbf{y}=\left(y_{0}, \ldots, y_{n-1}\right)$. Also, the restriction of $\beta$ over $W \times W$ is $\left.\beta\right|_{W \times W}\left(\left(x, x^{q}, \ldots, x^{q^{n-1}}\right),\left(y, y^{q}, \ldots, y^{q^{n-1}}\right)\right)=$ $\operatorname{Tr}_{q^{n} / q}(x y)$. Furthermore, with respect to the orthogonal complement operation $\perp$ defined by $\beta$ we have that

$$
\Gamma^{\perp}: x_{j}=\sum_{\ell=0}^{r-1} g_{j, \ell} x_{t_{\ell}} \quad j \notin\left\{t_{0}, \ldots, t_{r-1}\right\} .
$$


Then the Delsarte dual $\bar{U}_{\mathcal{C}}$ of $U_{\mathcal{C}}$ is the $\mathbb{F}_{q^{-}}$subspace $W+\Gamma^{\perp}$ of the quotient space $\mathbb{F}_{q^{n}}^{n} / \Gamma^{\perp}$ isomorphic to $U^{\prime}:=\left\langle W, \Gamma^{\perp}\right\rangle_{\mathbb{F}_{q}} \cap \Lambda^{\prime}$, where $\Lambda^{\prime}$ is the $\mathbb{F}_{q^{n-}}$ subspace of $\mathbb{F}_{q^{n}}^{n}$ of dimension $n-r$ represented by the following equations

$$
\Lambda^{\prime}: x_{t_{0}}=\ldots=x_{t_{r-1}}=0 .
$$

By identifying $\Lambda^{\prime}$ with $\mathbb{F}_{q^{n}}^{n-r}$, direct computations show that $U^{\prime}$ can be seen

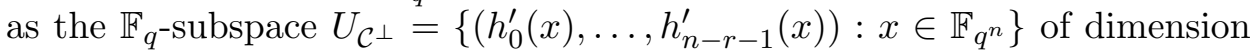
$n$ of $\mathbb{F}_{q^{n}}^{n-r}$, i.e. $U^{\prime}=U_{\mathcal{C}^{\perp}}$.

\section{Intersections of maximum $h$-scattered subspaces with hyperplanes}

This section is devoted to prove

Theorem 2.7 If $U$ is a maximum $h$-scattered $\mathbb{F}_{q}$-subspace of a vector space $V\left(r, q^{n}\right)$ of dimension $r n /(h+1)$, then for any $(r-1)$-dimensional $\mathbb{F}_{q^{n-}}$ subspace $W$ of $V\left(r, q^{n}\right)$ we have

$$
\frac{r n}{(h+1)}-n \leq \operatorname{dim}_{\mathbb{F}_{q}}(U \cap W) \leq \frac{r n}{(h+1)}-n+h .
$$

As we already mentioned, the theorem above is a generalization of [4, Theorem 4.2], which is the $h=1$ case of our result. In that paper, the number of hyperplanes meeting a 1-scattered subspace of dimension $\mathrm{rn} / 2$ in a subspace of dimension $r n / 2-n$ or $r n / 2-n+1$ has been determined as well. Subsequently to this paper, in [29] (see also [23] for the $h=2$ case), such values have been determined for every $h$.

\subsection{Preliminaries on Gaussian binomial coefficients}

The Gaussian binomial coefficient $\left[\begin{array}{l}n \\ k\end{array}\right]_{q}$ is defined as the number of the $k$ dimensional subspaces of the $n$-dimensional vector space $\mathbb{F}_{q}^{n}$. Hence

$$
\left[\begin{array}{l}
n \\
k
\end{array}\right]_{q}= \begin{cases}1 & \text { if } k=0 \\
\frac{\left(1-q^{n}\right)\left(1-q^{n-1}\right) \ldots\left(1-q^{n-k+1}\right)}{\left(1-q^{k}\right)\left(1-q^{k-1}\right) \ldots(1-q)} & \text { if } 1 \leq k \leq n \\
0 & \text { if } k>n\end{cases}
$$

Recall the following properties of the Gaussian binomial coefficients.

$$
\left[\begin{array}{l}
n \\
k
\end{array}\right]_{q}\left[\begin{array}{l}
k \\
j
\end{array}\right]_{q}=\left[\begin{array}{l}
n \\
j
\end{array}\right]_{q}\left[\begin{array}{l}
n-j \\
k-j
\end{array}\right]_{q}
$$




$$
\begin{gathered}
{\left[\begin{array}{l}
n \\
k
\end{array}\right]_{q}=\left[\begin{array}{c}
n \\
n-k
\end{array}\right]_{q} .} \\
\prod_{j=0}^{n-1}\left(1+q^{j} t\right)=\sum_{j=0}^{n} q^{j(j-1) / 2}\left[\begin{array}{l}
n \\
j
\end{array}\right]_{q} t^{j},
\end{gathered}
$$

Definition 5.1. The q-Pochhammer symbol is defined as

$$
(a ; q)_{k}=(1-a)(1-a q) \ldots\left(1-a q^{k-1}\right) .
$$

Theorem 5.2 ( $q$-binomial theorem [15, pg. 25, Exercise 1.3 (i)]).

$$
\begin{gathered}
(a b ; q)_{n}=\sum_{k=0}^{n} b^{k}\left[\begin{array}{l}
n \\
k
\end{array}\right]_{q}(a ; q)_{k}(b ; q)_{n-k}, \\
(a b ; q)_{n}=\sum_{k=0}^{n} a^{n-k}\left[\begin{array}{l}
n \\
k
\end{array}\right]_{q}(a ; q)_{k}(b ; q)_{n-k} .
\end{gathered}
$$

Corollary 5.3. In (19) and (20) put $a=q^{-n r / s}$ and $b=q^{n r / s-n}$ to obtain

$$
\begin{aligned}
& \left(q^{-n} ; q\right)_{s}=\sum_{j=0}^{s} q^{j(n r / s-n)}\left[\begin{array}{l}
s \\
j
\end{array}\right]_{q}\left(q^{-n r / s} ; q\right)_{j}\left(q^{n r / s-n} ; q\right)_{s-j}, \\
& \left(q^{-n} ; q\right)_{s}=q^{-n r} \sum_{j=0}^{s} q^{j n r / s}\left[\begin{array}{c}
s \\
j
\end{array}\right]_{q}\left(q^{-n r / s} ; q\right)_{j}\left(q^{n r / s-n} ; q\right)_{s-j},
\end{aligned}
$$

respectively.

The $l$-th elementary symmetric function of the variables $x_{1}, x_{2}, \ldots, x_{n}$ is the sum of all distinct monomials which can be formed by multiplying together $l$ distinct variables.

Definition 5.4. Denote by $\sigma_{k, l}$ the $l$-th elementary symmetric polynomial in $k+1$ variables evaluated in $1, q, q^{2}, \ldots, q^{k}$.

Lemma 5.5 ([6, Proposition 6.7 (b)]).

$$
\sigma_{k, l}=q^{l(l-1) / 2}\left[\begin{array}{c}
k+1 \\
l
\end{array}\right]_{q} .
$$

We will also need the following $q$-binomial inverse formula of Carlitz.

Theorem 5.6 ([7, special case of Theorem 2, pg. 897 (4.2) and (4.3)]). Suppose that $\left\{a_{k}\right\}_{k \geq 0}$ and $\left\{b_{k}\right\}_{k \geq 0}$ are two sequences of complex numbers. If $a_{k}=\sum_{j=0}^{k}(-1)^{j} q^{j(j-1) / 2}\left[\begin{array}{l}k \\ j\end{array}\right]_{q} b_{j}$, then $b_{k}=\sum_{j=0}^{k}(-1)^{j} q^{j(j+1) / 2-j k}\left[\begin{array}{l}k \\ j\end{array}\right]_{q} a_{j}$ and vice versa. 


\subsection{Double counting}

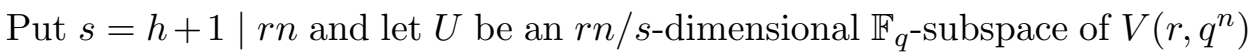

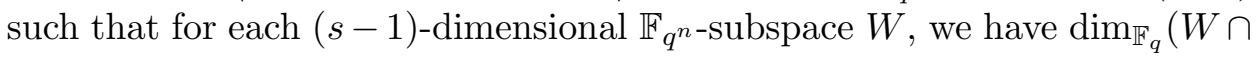
$U) \leq s-1$.

Let $h_{i}$ denote the number of $(r-1)$-dimensional $\mathbb{F}_{q^{n} \text {-subspaces meeting }}$ $U$ in an $\mathbb{F}_{q^{-}}$-subspace of dimension $i$. It is easy to see that

$$
h_{i}=0 \text { for } i<\frac{r n}{s}-n \text {. }
$$

In $\mathrm{PG}\left(V, \mathbb{F}_{q^{n}}\right)=\operatorname{PG}\left(r-1, q^{n}\right)$, the integer $h_{i}$ coincides with the number of hyperplanes $\operatorname{PG}\left(W, \mathbb{F}_{q^{n}}\right)$ such that $\operatorname{dim}_{\mathbb{F}_{q}}(W \cap U)=i$. Also, the number of hyperplanes is $\left(q^{r n}-1\right) /\left(q^{n}-1\right)$, which is the same as $\sum_{i} h_{i}$, thus

$$
\sum_{i} h_{i}\left(q^{n}-1\right)=q^{r n}-1
$$

For $k \in\{0,1, \ldots, s-1\}$ we can double count the set

$$
\begin{gathered}
\left\{\left(H,\left(P_{1}, P_{2}, \ldots, P_{k+1}\right)\right): H \text { is a hyperplane, } P_{1}, P_{2}, \ldots, P_{k+1} \in H \cap L_{U}\right. \\
\text { and } \left.\left\langle P_{1}, P_{2}, \ldots, P_{k+1}\right\rangle \cong \mathrm{PG}(k, q)\right\} .
\end{gathered}
$$

By Proposition 2.1 this gives

$$
\begin{gathered}
\sum_{i} h_{i}\left(\frac{q^{i}-1}{q-1}\right)\left(\frac{q^{i}-q}{q-1}\right) \ldots\left(\frac{q^{i}-q^{k}}{q-1}\right)= \\
\left(\frac{q^{r n / s}-1}{q-1}\right)\left(\frac{q^{r n / s}-q}{q-1}\right) \ldots\left(\frac{q^{r n / s}-q^{k}}{q-1}\right)\left(\frac{q^{(r-k-1) n}-1}{q^{n}-1}\right),
\end{gathered}
$$

or equivalently

\section{Lemma 5.7.}

$$
\begin{gathered}
\sum_{i} h_{i}\left(q^{n}-1\right)\left(q^{i}-1\right)\left(q^{i}-q\right)\left(q^{i}-q^{2}\right) \ldots\left(q^{i}-q^{k}\right)= \\
\left(q^{r n / s}-1\right)\left(q^{r n / s}-q\right)\left(q^{r n / s}-q^{2}\right) \ldots\left(q^{r n / s}-q^{k}\right)\left(q^{(r-k-1) n}-1\right) .
\end{gathered}
$$

Our aim is to prove

$$
A:=\sum_{i} h_{i}\left(q^{n}-1\right)\left(q^{i}-q^{n(r-s) / s}\right) \ldots\left(q^{i}-q^{n(r-s) / s+s-1}\right)=0 .
$$

This would clearly yield $h_{i}=0$, for $i>n(r-s) / s+s-1$, and hence Theorem 2.7. 


\subsection{Expressing $A$}

First for $k \in\{0, \ldots, s-1\}$ we will express

$$
\alpha_{k}:=\sum_{i} h_{i}\left(q^{n}-1\right) q^{k i} .
$$

Put $\beta_{0}:=\alpha_{0}=q^{r n}-1($ cf. (23)), and

$$
\beta_{k}:=\sum_{i} h_{i}\left(q^{n}-1\right)\left(q^{i}-1\right)\left(q^{i}-q\right) \ldots\left(q^{i}-q^{k-1}\right),
$$

where the values of $\beta_{k}$ are known due to Lemma 5.7.

Recall

$$
\sigma_{k, l}=\sum_{0 \leq i_{1}<\ldots<i_{l} \leq k} q^{i_{1}+\ldots+i_{l}} .
$$

Then it is easy to see that

$$
\alpha_{k}=\beta_{k}+\sum_{j=0}^{k-1}(-1)^{k-j-1} \alpha_{j} \sigma_{k-1, k-j}
$$

and hence, using also Lemma 5.5 .

$$
\beta_{k}=\sum_{j=0}^{k}(-1)^{k-j} q^{(k-j)(k-j-1) / 2} \alpha_{j}\left[\begin{array}{c}
k \\
k-j
\end{array}\right]_{q},
$$

or equivalently, by (17),

$$
\beta_{k} q^{-k(k-1) / 2}(-1)^{k}=\sum_{j=0}^{k} q^{j(j+1) / 2-j k}\left[\begin{array}{l}
k \\
j
\end{array}\right]_{q}(-1)^{j} \alpha_{j} .
$$

Then Theorem 5.6 applied to the sequences $\left\{a_{k}=\alpha_{k}\right\}_{k}$ and $\left\{b_{k}=\beta_{k} q^{-k(k-1) / 2}(-1)^{k}\right\}_{k}$ gives

$$
\alpha_{k}=\sum_{j=0}^{k}(-1)^{j} q^{j(j-1) / 2}\left[\begin{array}{l}
k \\
j
\end{array}\right]_{q} \beta_{j} q^{-j(j-1) / 2}(-1)^{j}=\sum_{j=0}^{k}\left[\begin{array}{l}
k \\
j
\end{array}\right]_{q} \beta_{j} .
$$

It is easy to see that

$$
A=\sum_{j=0}^{s}(-1)^{s-j} \alpha_{j} q^{(s-j) n(r-s) / s} \sigma_{s-1, s-j}
$$


and hence by Lemma 5.5

$$
A=\sum_{j=0}^{s}(-1)^{s-j} \alpha_{j} q^{(s-j) n(r-s) / s+(s-j)(s-j-1) / 2}\left[\begin{array}{c}
s \\
s-j
\end{array}\right]_{q} .
$$

By Lemma 5.7 we have

$$
\begin{gathered}
\beta_{k}=\left(q^{(r-k) n}-1\right) \prod_{j=0}^{k-1}\left(q^{r n / s}-q^{j}\right)= \\
\left(q^{(r-k) n}-1\right) q^{k(k-1) / 2}(-1)^{k} \prod_{j=0}^{k-1}\left(1-q^{r n / s-j}\right) .
\end{gathered}
$$

By 18 with $t=-q^{r n / s-k+1}$

$$
\prod_{j=0}^{k-1}\left(1-q^{r n / s-j}\right)=\prod_{j=0}^{k-1}\left(1-q^{r n / s-k+1} q^{j}\right)=\sum_{j=0}^{k} q^{j(j-1) / 2+r n j / s-(k-1) j}\left[\begin{array}{l}
k \\
j
\end{array}\right]_{q}(-1)^{j},
$$

thus

$$
\begin{gathered}
\beta_{k}=\sum_{j=0}^{k}\left(q^{(r-k) n}-1\right) q^{k(k-1) / 2} q^{j(j-1) / 2+r n j / s-(k-1) j}\left[\begin{array}{l}
k \\
j
\end{array}\right]_{q}(-1)^{j+k}= \\
\sum_{j=0}^{k}\left(q^{(r-k) n}-1\right) q^{(k-j)(k-j-1) / 2+r n j / s}\left[\begin{array}{c}
k \\
j
\end{array}\right]_{q}(-1)^{j+k}= \\
\sum_{t=0}^{k}\left(q^{(r-k) n}-1\right) q^{t(t-1) / 2+r n(k-t) / s}\left[\begin{array}{c}
k \\
t
\end{array}\right]_{q}(-1)^{t} .
\end{gathered}
$$

Hence by (24) and 25)

$$
A=\sum_{k=0}^{s} \sum_{j=0}^{k} \sum_{t=0}^{j}\left(q^{(r-j) n}-1\right) q^{t(t-1) / 2+r n(j-t) / s+(s-k) n(r-s) / s+(s-k)(s-k-1) / 2}\left[\begin{array}{c}
s \\
k
\end{array}\right]_{q}\left[\begin{array}{l}
k \\
j
\end{array}\right]_{q}\left[\begin{array}{l}
j \\
t
\end{array}\right]_{q}(-1)^{t+k+s} .
$$




\subsection{Proof of $A=0$}

Since $q$-binomial coefficients out of range are defined as zero, cf. (15), it is enough to prove that the following expression is zero:

$\sum_{k=0}^{s} \sum_{j=0}^{s} \sum_{t=0}^{s}\left(q^{r n-j n}-1\right) q^{r n j / s-r n t / s+(s-k) n(r-s) / s+\frac{1}{2}(s-k-1)(s-k)+\frac{1}{2}(t-1) t}\left[\begin{array}{l}s \\ k\end{array}\right]_{q}\left[\begin{array}{l}k \\ j\end{array}\right]_{q}\left[\begin{array}{l}j \\ t\end{array}\right]_{q}(-1)^{t+k}$.

It is clearly equivalent to prove $a_{s}=b_{s}$, where

$$
\begin{aligned}
& a_{s}=\sum_{j=0}^{s} q^{n r-n j} \sum_{k=0}^{s} \sum_{t=0}^{s} q^{r n j / s-r n t / s+(s-k) n(r-s) / s+\frac{1}{2}(s-k-1)(s-k)+\frac{1}{2}(t-1) t}\left[\begin{array}{l}
s \\
k
\end{array}\right]_{q}\left[\begin{array}{l}
k \\
j
\end{array}\right]_{q}\left[\begin{array}{l}
j \\
t
\end{array}\right]_{q}(-1)^{t+k}, \\
& b_{s}=\sum_{j=0}^{s} \sum_{k=0}^{s} \sum_{t=0}^{s} q^{r n j / s-r n t / s+(s-k) n(r-s) / s+\frac{1}{2}(s-k-1)(s-k)+\frac{1}{2}(t-1) t}\left[\begin{array}{l}
s \\
k
\end{array}\right]_{q}\left[\begin{array}{l}
k \\
j
\end{array}\right]_{q}\left[\begin{array}{l}
j \\
t
\end{array}\right]_{q}(-1)^{t+k} \text {. }
\end{aligned}
$$

\section{Proposition 5.8.}

$$
a_{s}=q^{n r}(-1)^{s}\left(q^{-n} ; q\right)_{s} .
$$

Proof. Clearly, it is enough to prove

$$
\begin{gathered}
(-1)^{s}\left(q^{-n} ; q\right)_{s}= \\
\sum_{j=0}^{s} q^{-n j} \sum_{k=0}^{s}(-1)^{k}\left[\begin{array}{l}
s \\
k
\end{array}\right]_{q}\left[\begin{array}{l}
k \\
j
\end{array}\right]_{q} q^{n r j / s+(s-k) n(r-s) / s+\frac{1}{2}(-k+s-1)(s-k)} \sum_{t=0}^{s} q^{-n r t / s+\frac{1}{2}(t-1) t}\left[\begin{array}{l}
j \\
t
\end{array}\right]_{q}(-1)^{t},
\end{gathered}
$$

where by 18

$$
\sum_{t=0}^{s} q^{-n r t / s+\frac{1}{2}(t-1) t}\left[\begin{array}{l}
j \\
t
\end{array}\right]_{q}(-1)^{t}=\left(q^{-n r / s} ; q\right)_{j},
$$

thus the triple sum can be reduced to

$$
\sum_{j=0}^{s} q^{n r j / s-n j}\left(q^{-n r / s} ; q\right)_{j} \sum_{k=0}^{s}(-1)^{k}\left[\begin{array}{l}
s \\
k
\end{array}\right]_{q}\left[\begin{array}{l}
k \\
j
\end{array}\right]_{q} q^{(s-k) n(r-s) / s+\frac{1}{2}(-k+s-1)(s-k)} .
$$

By (16) and (17) this can be written as

$$
\sum_{j=0}^{s} q^{n r j / s-n j}\left(q^{-n r / s} ; q\right)_{j}\left[\begin{array}{l}
s \\
j
\end{array}\right]_{q} \sum_{k=0}^{s}(-1)^{k}\left[\begin{array}{l}
s-j \\
s-k
\end{array}\right]_{q} q^{(s-k) n(r-s) / s+\frac{1}{2}(-k+s-1)(s-k)},
$$


where again by 18

$$
\begin{gathered}
\sum_{k=0}^{s}(-1)^{k}\left[\begin{array}{l}
s-j \\
s-k
\end{array}\right]_{q} q^{(s-k) n(r-s) / s+\frac{1}{2}(-k+s-1)(s-k)}= \\
(-1)^{s} \sum_{z=0}^{s}(-1)^{z}\left[\begin{array}{c}
s-j \\
z
\end{array}\right]_{q} q^{z n(r-s) / s+z(z-1) / 2}= \\
(-1)^{s}\left(q^{n r / s-n} ; q\right)_{s-j} .
\end{gathered}
$$

By (26) we have

$$
(-1)^{s} \sum_{j=0}^{s} q^{j(n r / s-n)}\left[\begin{array}{l}
s \\
j
\end{array}\right]_{q}\left(q^{-n r / s} ; q\right)_{j}\left(q^{n r / s-n} ; q\right)_{s-j},
$$

which by 21) equals $(-1)^{s}\left(q^{-n} ; q\right)_{s}$.

\section{Proposition 5.9.}

$$
b_{s}=q^{n r}(-1)^{s}\left(q^{-n} ; q\right)_{s} .
$$

Proof. As before, $b_{s}$ can be written as

$$
q^{n r}(-1)^{s} \sum_{j=0}^{s} q^{j n r / s-n r}\left[\begin{array}{l}
s \\
j
\end{array}\right]_{q}\left(q^{-n r / s} ; q\right)_{j}\left(q^{n r / s-n} ; q\right)_{s-j} .
$$

Then the assertion follows from 22 .

\section{References}

[1] S. Ball, A. Blokhuis And M. Lavrauw: Linear $(q+1)$-fold blocking sets in PG(2, $\left.q^{4}\right)$, Finite Fields Appl. 6 (4) (2000), 294-301.

[2] D. Bartoli, B. Csajbók, G. Marino and R. Trombetti: Evasive subspaces, arXiv:2005.08401.

[3] D. Bartoli, M. Giulietti, G. Marino and O. Polverino: Maximum scattered linear sets and complete caps in Galois spaces, Combinatorica 38(2) (2018), 255-278.

[4] A. Blokhuis and M. Lavrauw: Scattered spaces with respect to a spread in PG(n,q), Geom. Dedicata 81 (2000), 231-243. 
[5] G. Bonoli and O. Polverino: $\mathbb{F}_{q}$-linear blocking sets in $\operatorname{PG}\left(2, q^{4}\right)$, Innov. Incidence Geom. 2 (2005): 35-56.

[6] P. J. Cameron: Notes on Counting: An Introduction to Enumerative Combinatorics, Cambridge University Press 2017.

[7] L. Carlitz: Some inverse relations, Duke Mathematical Journal 40 (1973), no. 4, 893-901.

[8] B. Csajbók, G. Marino and O. Polverino: Classes and equivalence of linear sets in PG(1, $\left.q^{n}\right)$, J. Combin. Theory Ser. A 157 (2018), $402-426$.

[9] B. Csajbók, G. Marino and O. Polverino: A Carlitz type result for linearized polynomials, Ars Math. Contemp. 16(2) (2019), 585608.

[10] B. Csajbók, G. Marino, O. Polverino and Y. Zhou: Maximum Rank-Distance codes with maximum left and right idealisers, Discrete Math. 343(9) (2020).

[11] B. Csajbók, G. Marino, O. Polverino and F. Zullo: Maximum scattered linear sets and MRD-codes, J. Algebraic Combin. 46 (2017), $1-15$.

[12] B. Csajbók and C. Zanella: On the equivalence of linear sets, Des. Codes Cryptogr. 81 (2016), 269-281.

[13] P. Delsarte: Bilinear forms over a finite field, with applications to coding theory, J. Combin. Theory Ser. A 25 (1978), 226-241.

[14] E. Gabidulin: Theory of codes with maximum rank distance, Problems of information transmission, 21(3) (1985), 3-16.

[15] G. Gasper and M. Rahman: Basic Hypergeometric Series (Encyclopedia of Mathematics and its Applications), Cambridge University Press (2004).

[16] B. HupperT: Endliche Gruppen, volume 1. Springer BerlinHeidelberg-New York, 1967.

[17] M. Lavrauw: Scattered spaces in Galois Geometry, Contemporary Developments in Finite Fields and Applications, 2016, 195-216. 
[18] M. Lavrauw and G. Van de Voorde: Field reduction and linear sets in finite geometry, In: Topics in Finite Fields, AMS Contemporary Math, vol. 623, pp. 271--293. American Mathematical Society, Providence (2015).

[19] G. Lunardon: MRD-codes and linear sets, J. Combin. Theory Ser. A 149 (2017), 1-20.

[20] G. Lunardon, P. Polito and O. Polverino: A geometric characterisation of linear k-blocking sets, J. Geom. 74 (1-2) (2002), 120-122.

[21] G. Lunardon and O. Polverino: Translation ovoids of orthogonal polar spaces, Forum Math. 16 (2004), 663-669.

[22] G. Lunardon, R. Trombetti and Y. Zhou: On kernels and nuclei of rank metric codes, J. Algebraic Combin. 46 (2017), 313-340.

[23] V. Napolitano and F. Zullo: Codes with few weights arising from linear sets, arXiv:2002.07241.

[24] O. Polverino: Linear sets in finite projective spaces, Discrete Math. 310(22) (2010), 3096-3107.

[25] A. Ravagnani: Rank-metric codes and their duality theory, Des. Codes Cryptogr. 80(1) (2016), 197-216.

[26] B. Segre: Teoria di Galois, fibrazioni proiettive e geometrie non Desarguesiane, Ann. Mat. Pura Appl. 64 (1964), 1-76.

[27] J. Sheekey: MRD codes: constructions and connections, Combinatorics and finite fields: Difference sets, polynomials, pseudorandomness and applications, Radon Series on Computational and Applied Mathematics, K.-U. Schmidt and A. Winterhof (eds.).

[28] J. Sheekey and G. VAn de Voorde: Rank-metric codes, linear sets and their duality, Des. Codes Cryptogr. 88 (2020), 655 - 675.

[29] G. Zini And F. Zullo: Scattered subspaces and related codes, submitted.

[30] F. Zullo: Linear codes and Galois geometries, Ph.D thesis, Università degli Studi della Campania "Luigi Vanvitelli". 
Bence Csajbók

MTA-ELTE Geometric and Algebraic Combinatorics Research Group

ELTE Eötvös Loránd University, Budapest, Hungary

Department of Geometry

1117 Budapest, Pázmány P. stny. 1/C, Hungary

csajbokb@cs.elte.hu

Giuseppe Marino

Dipartimento di Matematica e Applicazioni "Renato Caccioppoli"

Università degli Studi di Napoli "Federico II",

Via Cintia, Monte S.Angelo I-80126 Napoli, Italy

giuseppe.marino@unina.it

Olga Polverino and Ferdinando Zullo

Dipartimento di Matematica e Fisica,

Università degli Studi della Campania "Luigi Vanvitelli",

I- 81100 Caserta, Italy

olga.polverino@unicampania.it,

ferdinando.zullo@unicampania.it 